\title{
Microbial Colonization in Adulthood Shapes the Intestinal Macrophage Compartment
}

\author{
Franziska Schmidt, ${ }^{a, b}$ Katja Dahlke, ${ }^{c}$ Arvind Batra, Jacqueline Keye, ${ }^{a, b}$ \\ Hao Wu, ,ab Marie Friedrich, ${ }^{\mathrm{a}, \mathrm{b}}$ Rainer Glauben, ${ }^{\mathrm{a}}$ Christiane Ring, ${ }^{\mathrm{c}}$ \\ Gunnar Loh, ${ }^{,}$Monika Schaubeck, ${ }^{\mathrm{d}}$ Hubert Hackl,e Zlatko Trajanoski, \\ Michael Schumann, ${ }^{a}$ Anja A. Kühl, ${ }^{a}$ Michael Blaut, ${ }^{,}$Britta Siegmund ${ }^{a}$
}

\begin{abstract}
aMedizinische Klinik für Gastroenterologie, Infektiologie und Rheumatologie, Charité - Universitätsmedizin Berlin, corporate member of Freie Universität Berlin, Humboldt-Universität zu Berlin, and Berlin Institute of Health, Berlin, Germany ${ }^{b}$ Fachbereich Biologie, Chemie, Pharmazie, Freie Universität Berlin, Berlin, Germany 'Department of Gastrointestinal Microbiology, German Institute of Human Nutrition Potsdam-Rehbruecke, Nuthetal, Germany ${ }^{\mathrm{d} N e u r o i m m u n o l o g y, ~ M a x-P l a n c k-I n s t i t u t e ~ o f ~ N e u r o b i o l o g y, ~ P l a n e g g-M a r t i n s r i e d, ~ G e r m a n y ~}{ }^{\mathrm{e} B i o c e n t e r}$, Division of Bioinformatics, Medical University of Innsbruck, Innsbruck, Austria
\end{abstract}

Corresponding author: Britta Siegmund, MD, Medizinische Klinik für Gastroenterologie, Infektiologie und Rheumatologie, Charité - Universitätsmedizin Berlin, Hindenburgdamm 30, 12200 Berlin, Germany. Tel: 49-30-450-514322, Fax: 49-30-450514990; Email: britta.siegmund@charite.de

\begin{abstract}
Background and Aims: Contact with distinct microbiota early in life has been shown to educate the mucosal immune system, hence providing protection against immune-mediated diseases. However, the impact of early versus late colonization with regard to the development of the intestinal macrophage compartment has not been studied so far.

Methods: Germ-free mice were colonized with specific-pathogen-free [SPF] microbiota at the age of 5 weeks. The ileal and colonic macrophage compartment were analysed by immunohistochemistry, flow cytometry, and RNA sequencing 1 and 5 weeks after colonization and in age-matched SPF mice, which had had contact with microbiota since birth. To evaluate the functional differences, dextran sulfate sodium [DSS]-induced colitis was induced, and barrier function analyses were undertaken. Results: Germ-free mice were characterized by an atrophied intestinal wall and a profoundly reduced number of ileal macrophages. Strikingly, morphological restoration of the intestine occurred within the first week after colonization. In contrast, ileal macrophages required 5 weeks for complete restoration, whereas colonic macrophages were numerically unaffected. However, following DSS exposure, the presence of microbiota was a prerequisite for colonic macrophage infiltration. One week after colonization, mild colonic inflammation was observed, paralleled by a reduced inflammatory response after DSS treatment, in comparison with SPF mice. This attenuated inflammation was paralleled by a lack of TNF $\alpha$ production of LPS-stimulated colonic macrophages from SPF and colonized mice, suggesting desensitization of colonized mice by the colonization itself. Conclusions: This study provides the first data indicating that after colonization of adult mice, the numeric, phenotypic, and functional restoration of the macrophage compartment requires the presence of intestinal microbiota and is time dependent.
\end{abstract}

Abbreviations: GF, germ-free; SPF, specific-pathogen-free; COL, colonized at Week 5 of age; DSS, dextran sulfate sodium; NKT, natural killer T; LPS, lipopolysaccharide; IBD, inflammatory bowel disease; LPMCs, lamina propria mononuclear cells. 
Key Words: Microbial colonization; intestinal macrophages; DSS colitis

\section{Introduction}

The incidence of inflammatory bowel disease [IBD] has been rising over recent decades. ${ }^{1}$ While it is known that $~ 25 \%$ of IBD cases can be explained genetically, ${ }^{2}$ this recent increase in incidence cannot be driven by genetic factors, but rather by environmental changes.

The intestinal microbiota composition strongly depends on the environment and is a key factor in maintaining intestinal homeostasis. ${ }^{3-6}$ Furthermore, the commensal microbiota equally contributes to enterocyte maturation and angiogenesis, and consequently to intestinal barrier function. ${ }^{7,8}$ Whether the time point of intestinal colonization in life matters for the composition of the intestinal immune cells has so far only partially been addressed.

That an early exposure to distinct microbiota might play a role in local immune cell maturation is indicated by data about children growing up in a farm environment revealing a decreased risk for developing asthma..$^{9,10}$ In line, exposure to antibiotics within the first year of life has been linked to an increased risk of developing IBD. ${ }^{11,12}$ The so-called 'window of opportunity', the critical time for microbial contact impacting on the development of the mucosal immune system was previously studied with germ-free [GF] mice. The time point of colonization after birth affects the composition of the natural killer T [NKT] cell compartment, inducing a more proinflammatory state when colonization occurs later in life [at the age of 5 weeks]. ${ }^{13}$ Furthermore, GF mice have previously been characterized by high immunoglobulin $\mathrm{E}[\mathrm{IgE}]$ levels. ${ }^{14-17} \mathrm{~A}$ recent study adds to these data by providing evidence that a distinct level of microbiota diversity following birth forms a prerequisite for inhibition of $\operatorname{IgE}$ production. ${ }^{15}$ Together, these clinical and experimental data fit into the hygiene hypothesis claiming that exposure to bacteria early in life mediates a protection from autoimmune diseases later on. ${ }^{18}$

Apart from these findings, there is still a need to explore whether the time point of colonization impacts the composition of the intestinal myeloid cells. In addition, little is known about the impact of the luminal microbiota on the formation of the macrophage compartment, and the subsequent functional consequences under healthy and inflammatory conditions. In intestinal homeostasis, anti-inflammatory macrophages are localized underneath the epithelium and play a decisive role in maintaining the intestinal homeostasis by removal of pathogens without an induction of inflammatory responses of lymphocytes. Intestinal macrophages possess tolerance against foreign material by downregulated expression of receptors for recognition, ${ }^{19}$ or by downregulation of adaptor molecules associated with pattern recognition. ${ }^{20-22}$ Food antigens or commensal bacteria are recognized by intestinal macrophages, and processed antigens are presented without co-stimulatory molecules. ${ }^{23}$ When considering the function of intestinal macrophages, one has to differentiate between those in the small intestine and those in the colon. While macrophages in the small intestine are more involved in processing food antigens, colonic macrophages have to deal with a higher bacterial load and the associated metabolites that can directly influence macrophage function. For instance, butyrate, a short-chain fatty acid produced in large quantities by commensal bacteria, is known for its regulatory function, in macrophages as well as in epithelial cells, of inhibiting histone deacetylases. ${ }^{24}$

In the lamina propria of IBD patients, an accumulation of proinflammatory macrophages expressing costimulatory molecules and macrophage-activating receptors is observed. Functional data indicate that these pro-inflammatory macrophages directly disturb barrier function and thus further enhance intestinal inflammation..$^{25}$ In addition, treatment with TNF $\alpha$ antibodies in IBD has been associated with apoptosis of pro-inflammatory [M1] macrophages and a parallel increase in anti-inflammatory [M2] macrophages..$^{26-28}$ These data underline that the balance of pro- and anti-inflammatory macrophages is crucial in maintaining intestinal homeostasis. ${ }^{29}$

There are limited data in the literature concerning how the microbiota influences the intestinal macrophage compartment. For instance, lipopolysaccharides [LPSs] have been shown to induce IL-10 expression in intestinal macrophages from specific-pathogenfree [SPF] but not GF mice, ${ }^{30}$ hence, indicating that the intestinal microbiota regulates the crosstalk between macrophages and innate lymphoid cells that mediates intestinal immune homeostasis. ${ }^{4}$

Macrophages in many tissues are tissue-derived cells and are present from birth. ${ }^{31,32}$ Data indicate that in the neonatal period, the colonic macrophage compartment develops from embryonic precursor cells, which proliferate locally afterwards. Around the time of weaning, these precursor cells are replaced by infiltrating monocytes. These Ly6 $\mathrm{C}^{\text {high }}$ monocytes then differentiate into mature intestinal macrophages. ${ }^{33}$ These data suggest that the time point of colonization is relevant for the composition and function of the macrophage compartment. However, very recent studies demonstrate that, in the intestine, a tissue-derived self-renewing macrophage population exists. $^{34,35}$

Monocytes infiltrating the tissue as well as macrophages possess the remarkable plasticity to differentiate further, following stimulation with either defined cytokines and/or microbial molecules. ${ }^{36}$ This feature is a prerequisite for the potency of these cells to adapt their phenotype to a specific milieu. ${ }^{37}$ For example, colitis triggers monocytes to differentiate into pro-inflammatory myeloid cells in the colon. ${ }^{38,39}$ However, it is unclear whether this adaptation of the macrophage compartment can occur throughout life and whether or not it requires colonization starting from birth.

To fill this gap, we here addressed the question of whether and how the macrophage compartment is restored when colonization occurs later in life.

\section{Material and Methods}

\subsection{Animals}

Male wild type [WT] $\mathrm{C} 3 \mathrm{H} / \mathrm{HeOuJ}$ mice at the age of 5, 6, 9, and 10 weeks were used for the experiments. Germ-free, SPF, and GF-born and at the age of 5 weeks colonized [COL] mice were maintained in positive-pressure isolators [Metall + Plastic, Radolfzell, Germany] under a $12 \mathrm{~h}$ light-dark cycle. The mice had free access to irradiated standard chow [Altromin fortified type 1310; Altromin, Lage, Germany] and autoclaved distilled water. The animal experiments were approved by the Animal Welfare Committee of the state of Brandenburg, Germany.

\subsection{Chemicals}

If not indicated otherwise, all chemicals and media were obtained from Sigma-Aldrich Chemie GmbH [Taufkirchen, Germany]. 


\subsection{Colonization of GF mice with SPF microbiota}

A suspension of $200 \mathrm{mg}$ of faeces from SPF mice in $10 \mathrm{~mL}$ drinking water was made up. Each GF mouse received $100 \mu \mathrm{l}$ of this suspension by oral gavage at the age of 5 weeks. Successful colonization after inoculation was validated by inspecting the faeces via microscopy after Gram-staining and by high-throughput $16 \mathrm{~S}$ ribosomal RNA [rRNA] gene amplicon sequencing of caecal content after culling.

\subsection{High-throughput $16 \mathrm{~S}$ rRNA gene sequencing}

Sequencing of bacterial 16S rRNA amplicon libraries was performed by CeMeT GmbH [Tübingen, Germany]. DNA was isolated from snap-frozen caecal contents according to a previously published protocol based on mechanical lysis and purification by DNA precipitation. ${ }^{40}$ The variable regions V3/V4 were amplified using primers 341F: 5'-CCTACGGGNGGCWGCAG-3' and 785R: 5'-GACTACHVGGGTATCTAATCC-3' ${ }^{41}$ Amplicons were purified using magnetic beads and sequenced using a MiSeq sequencer [Illumina Inc., San Diego, CA, USA]. Sequences were further processed using Integrated Microbial Next-Generation Sequencing [IMNGS] ${ }^{42}$ In brief, sequences were demultiplexed and trimmed, and sequences with $<380$ and $>420$ nucleotides were excluded from the analysis. Operational taxonomic units [OTUs] were clustered at $97 \%$ sequence similarity, and only those with a relative abundance of $>0.5 \%$ were kept. All details of the analysis and the scripts are available online [https://lagkouvardos.github.io/Rhea/]. ${ }^{43}$

The $16 \mathrm{~S}$ rRNA gene sequencing data are available under accession number SRP131846 [https://www.ncbi.nlm.nih.gov/sra/ SRP131846] in the NCBI Sequence Read Archive.

\subsection{Dextran sulfate sodium colitis and scoring}

Mice were exposed to dextran sulfate sodium [DSS, 36 000-50 000 Da, MP Biomedicals, LLC, Santa Ana, USA] for 4 days via the drinking water in the concentrations as indicated. After an additional 1 or 3 days with regular drinking water, the mice were killed and their organs and faeces were collected for further analyses. The disease score and histomorphological signs of inflammation were evaluated as described previously. ${ }^{44,45}$

\subsection{Immunohistochemistry and immunofluorescence}

For immunohistochemistry, formalin-fixed intestinal tissue sections were subjected to a heat-induced epitope retrieval step prior to incubation with anti-cleaved caspase 3 [Asp175, Cell Signaling Technology, Leiden, Netherlands], followed by detection using the Labelled-Streptavidin-Biotin [LSAB] method employing the Dako REAL ${ }^{\text {TM }}$ Detection System, and Alkaline Phosphatase/RED, Rabbit/Mouse [Agilent Technologies, Santa Clara, CA, USA]. Nuclei were counterstained with hematoxylin [Merck KGaA, Darmstadt, Germany]. For the detection of macrophages, sections were subjected to protein-induced epitope retrieval employing protease prior to incubation with anti-F4/80 [BM8, eBioscience, USA] followed by detection using the LSAB-method. For the detection of anti-inflammatory macrophages, sections were incubated with anti-Ym1 [EPR15263, Abcam]. For detection, Alexa488-labelled secondary antibodies [Thermo Fisher Scientific] were used. Nuclei were counterstained with 4',6-Diamidin-2-phenylindol [DAPI]. For the detection of claudin 1, sections were subjected to a heatinduced epitope-retrieval step prior to incubation with anti-claudin 1 [polyclonal rabbit, Thermo Fisher Scientific], followed by detection employing the EnVision Detection System, Peroxidase/DAB, Rabbit [Agilent Technologies, USA]. Nuclei were counterstained with haematoxylin.
Negative controls were performed by omitting the primary antibodies. Images were acquired using the AxioImager $\mathrm{Z} 1$ microscope [Carl Zeiss MicroImaging GmbH, Jena, Germany]. All evaluations were performed blinded. Positive cells were quantified in at least five high-power fields $\left[1 \mathrm{hpf}=0.237 \mathrm{~mm}^{2}\right]$. Claudin 1 expression was scored semiquantitatively ranging from 0 [no expression] to 3 [high expression].

\subsection{Mucin staining and measurement of mucosa thickness}

Periodic acid Schiff-reaction/alcian blue [PAS/AB] mucin staining of Carnoy's-fixed intestinal tissue sections was performed as previously described. ${ }^{46}$ For mucosa thickness, $\sim 30$ crypts or villi per section were analysed using an Eclipse E600 microscope [Nikon GmbH, Düsseldorf, Germany] with Lucia G software version 4.51 [Laboratory imaging s.r.o.; Microsoft, Munich, Germany]. Images were captured with an MV-1500 digital camera [Nikon GmbH].

\subsection{Isolation and stimulation of mononuclear cells from the lamina propria}

For the isolation of lamina propria mononuclear cells [LPMCs], the colon or ileum [10 $\mathrm{cm}$ distal part] was cut into small pieces and then incubated twice with $5 \mathrm{mM}$ ETDA/HBSS. This was followed by enzymatic digestion $(0.44 \mathrm{mg} / \mathrm{mL}$ Collagenase D and $20 \mu \mathrm{g} / \mathrm{mL}$ DNase I in Roswell Park Memorial Institute medium [RPMI]). After filtering, the LPMCs were purified by Percoll ${ }^{\mathrm{TM}}$ [GE Healthcare, Little Chalfont, UK] density gradient centrifugation.

For analysing intracellular TNF $\alpha$-expression, LPMCs were stimulated with $1 \mu \mathrm{g} / \mathrm{mL}$ LPS [from Escherichia coli O111:B4] for $3 \mathrm{~h}$; for the last $2 \mathrm{~h}, 5 \mu \mathrm{g} / \mathrm{mL}$ brefeldin A was added.

\subsection{Flow cytometry}

The following antibodies [clones] were used to stain LPMCs: anti-F4/80-eFluor450 [BM8], anti-CD11c-PercpCy5.5 [N418], anti-MHCII-FITC [M5/114.15.2], anti-CD11b-APC/Cy7 [M1/70; all eBioscience, San Diego, USA], and anti-Ly6C-PE [HK1.4; BioLegend, San Diego, USA]. Live and dead cells were discriminated using LIVE/DEAD ${ }^{\circledR}$ Fixable Aqua Dead Cell Stain Kit [Thermo Fisher Scientific]. Samples were measured using a FACS CantoII device [BD Biosciences, Heidelberg, Gemany] and were analysed using FlowJo Software V8.8.7 [FlowJo, LLC, Ashland, USA]. Frequencies and cell numbers counted in the Neubauer chamber served to calculate absolute cell counts. The gating is shown in Supplementary Figure 1.

\subsection{Cytokine expression of ex vivo tissue culture}

Approximately $1 \mathrm{~cm}^{2}$ of colon tissue was weighed and cultured for $20 \mathrm{~h}$ in serum-free RPMI 1640 medium [100 U/mL penicillin, $100 \mu \mathrm{g} / \mathrm{mL}$ streptomycin, $2 \mathrm{mM} \mathrm{L}$-glutamine]. Cytokines in the supernatant were determined with the Cytometric Bead Array Mouse Inflammation Kit [BD Biosciences]. Data were assessed applying a FACS CantoII device and were analysed using FCAP software V1 [BD Biosciences]. Data were expressed in relation to tissue weight.

\subsection{Intestinal barrier function}

Colon tissue was mounted in a miniaturized Ussing chamber [area $\left.0.049 \mathrm{~cm}^{2}\right]$ as described previously. ${ }^{47}$ Results were presented as transmural wall resistance $\left[R^{t}\right]$, subepithelial resistance $\left[R^{s}\right]$, and epithelial resistance $\left[R^{\text {epi }}, R^{t}\right.$ minus $\left.R^{s}\right]$. Paracellular permeability was determined by measuring mannitol flux [mucosal-to-serosal] as 
previously described. ${ }^{47}$ Transcellular transport was determined by horseradish peroxidase flux. Horseradish peroxidase type VI was applied at a final concentration of $18.1 \mu \mathrm{M}$ to the mucosal side. Serosal fractions were sampled five times each $30 \mathrm{~min}$ and analysed by a QuantaBlu ${ }^{\mathrm{TM}}$ Fluorogenic Peroxidase Substrate Kit [Thermo Fisher Scientific].

\subsection{RNA sequencing}

Total RNA was isolated from whole tissue by the use of Qiazol lysis reagent [Qiagen, Hilden Germany] and was submitted to transcriptome analysis for the purpose of gene-expression profiling, using the QuantSeq 3' mRNA-Seq Library Prep Kit for Ion Torrent [Lexogen, Vienna Biocenter] for generation of 3' polyA tag mRNA libraries. Quality-validated, barcoded libraries were multiplexed, templated, and sequenced using the Ion OneTouch ${ }^{\mathrm{TM}}$ and the Ion Proton ${ }^{\mathrm{TM}}$ systems [Ion Torrent, Thermo Fisher Scientific]. Library preparation and sequencing was carried out at the GenomeSeq Core of the Medical University of Innsbruck.

Base calling and barcode filtering were performed using Torrent Suite [5.8.0]. From single-end reads [FASTQ files], adopters were removed and reads were cropped at $200 \mathrm{bp}$ using Trimmomatic $[0.36] .{ }^{48}$ Sequencing quality was checked using FastQC [0.11.5]. Reads were mapped using STAR aligner $(2.5 .3 \mathrm{a}]^{49}$ onto the mouse genome version mm10 [UCSC] with RefGene annotation and indexed with $200 \mathrm{bp}$ splice junction overhang. HTSeq ${ }^{50}$ were used to quantify gene raw counts. DESeq $2^{51}$ within the R environment [3.4.1] were used to identify differentially expressed genes using a negative binomial distribution filtered for genes with normalized mean counts $>10$ across the compared samples. $p$-values were adjusted for multiple testing based on the false discovery rate [FDR] using the Benjamini-Hochberg method. Genes were considered differentially expressed with $>1.5$ fold change and adjusted $p[$ FDR $]<0.1$. Counts were normalized by library-size factors and regularized log transformed for subsequent analyses. Gene set enrichment analysis was performed using GSEA software ${ }^{52,53}$ and a gene ontology gene set downloaded from http://ge-lab.org/gskb/. Gene sets with a normalized enrichment score [NES] of $>2.0$ or $<-2.0$ [and FDR $<0.1$ ] were considered to be highly enriched. Heat maps were generated using Genesis [1.8.1]..54 The data have been deposited in NCBI's Gene Expression Omnibus ${ }^{55}$ and are accessible through GEO Series accession number GSE115202 [https://www.ncbi.nlm.nih.gov/geo/query/ acc.cgi?acc=GSE115202].

\subsection{Statistical analysis}

Statistical analyses were performed with GraphPad PRISM software, version 6.0 [GraphPad Software, San Diego, CA] using analysis of variance [ANOVA] followed by pairwise comparison testing [HolmSidak test]. The histological disease score data and barrier data were tested for statistical significance using a two-sided Mann-WhitneyU-Test. The survival data were analysed with the Log-Rank-Test. Data are presented as single values and mean. $p$-values of $<0.05$ were considered to be statistically significant. For RNA analyses, an adjusted $p$-value of $<0.1$ was considered to be statistically significant.

\section{Results}

\subsection{Colonization of germ-free born mice restored the structure of the gut}

Germ-free mice were colonized with SPF microbiota at the age of 5 weeks. This time point was chosen to analyse the effect of first microbial contact after weaning. The effects of colonization preweaning, here at birth, was assessed using SPF mice. To determine the impact on intestinal structure and composition of the myeloid compartment, mice were analysed 1 week and 5 weeks after colonization, as well in age-matched GF and SPF mice [Figure 1A].

Germ-free mice presented an atrophied ileal mucosa in comparison with SPF mice at both time points analysed. The microbial colonization restored the structural differences by 1 week after colonization. Conversely, the colon mucosa of GF mice at Week 10 did not differ from that of SPF and COL mice. In addition, in COL mice, colon mucosa thickness adapted within 1 week of colonization [Figure 1B, C]. The expression of the tight junction protein claudin 1 was significantly decreased in the ileum of GF mice at both ages. Remarkably, the expression normalized within 1 week when GF mice were colonized with microbiota [Figure 1D, E].

To assess the microbial status of COL mice over time in comparison with that of age-matched SPF mice, 16S rRNA gene sequencing was performed. One week after colonization, the caecal content of COL mice presented with a lower richness and Shannon effective species count [Figure 2A] compared with that of COL mice at 10 weeks and SPF mice at either time point. We found no difference in $\beta$-diversity of SPF mice at 6 or 10 weeks of age. The bacterial community profiles of COL mice at 6 and 10 weeks were clearly separated from those of SPF mice, which grouped closely together [Figure 2B]. These differences in community structure were reflected in the relative abundance of the bacterial families [Figure 2C]. The microbiota adapted in a time-dependent manner after colonization, but did not reach the status of SPF mice in regard to $\beta$-diversity.

Remarkably, colonization induced an inflammatory response in the colon [Figure 2D/E], but not in the ileum [data not shown]. A subset of COL mice presented discrete histologic signs of colonic inflammation shortly after colonization, which disappeared at 10 weeks of age [Figure 2D, E]. This was characterized by a marginal immune cell infiltration (of macrophages and CD4 ${ }^{+} \mathrm{T}$ cells [Supplementary Figure 2]) into the colonic mucosa, but not by epithelial damage, emphasizing the role of the intestinal microbiota as stimulus for immune cell infiltration into the lamina propria.

\subsection{Time-dependent restoration of the macrophage compartment after colonization}

We hypothesized that the macrophage compartment is influenced by microbial colonization and develops in a time-dependent manner following colonization.

For this purpose, the intestinal myeloid cell compartments in the ileum and colon were evaluated over time. The number of $\mathrm{F} 4 / 80^{+} \mathrm{CD} 11 \mathrm{~b}^{+}$cells [reflecting the number of macrophages] in the ileum was significantly higher in SPF than in GF mice [Figure 3A]. Following colonization, full restoration of these cells required at the longest 5 weeks in the ileum [Figure 1A and 3A]. The frequency of MHCII and CD11c of F4/80+ CD11 $b^{+}$macrophages was increased in SPF compared with GF and COL mice at 5 weeks of age, which was not observed at Week 10.

CD11 ${ }^{+}$Ly6C $\mathrm{C}^{+}$cells [reflecting monocytes] presented with an increase in number at 5 weeks after colonization. One week after colonization, the numbers of recently infiltrated monocytes in the ileum equalled the number in GF mice. Analyses of macrophages and monocytes in the colon did not reveal numeric differences between GF, SPF, or COL mice, at either time point. The frequency of CD11 ${ }^{+}$ macrophages did not differ between the groups, but a higher number 
A

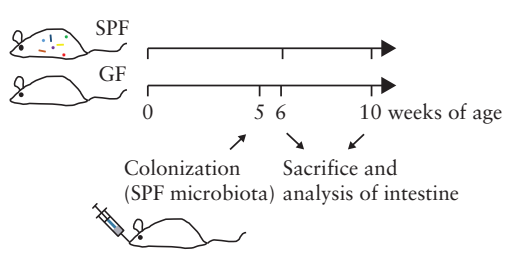

B

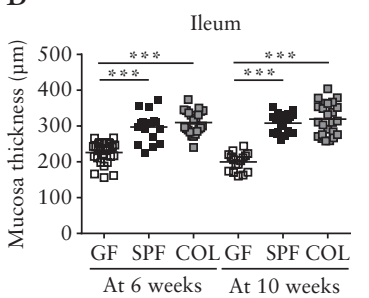

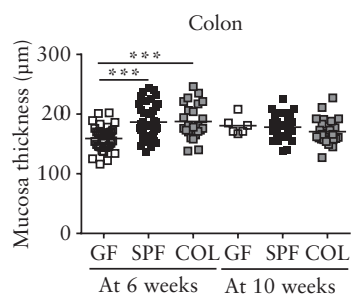

$\mathrm{COL}$

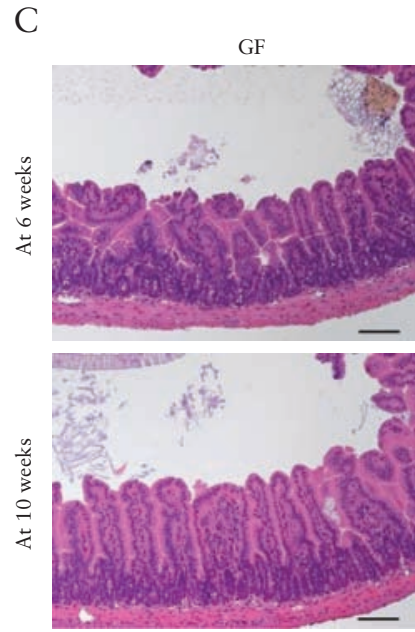

SPF
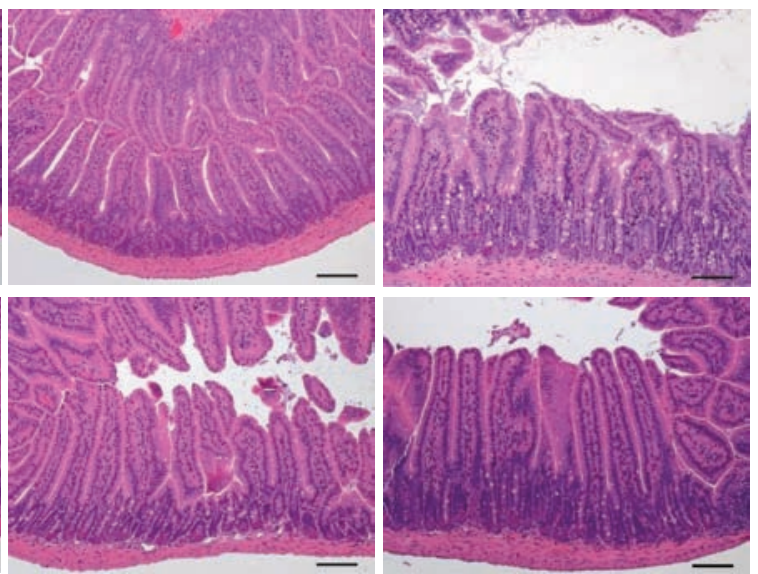

$\mathrm{D}$

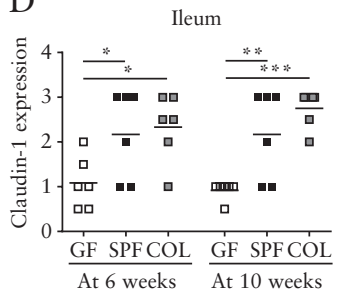

Colon

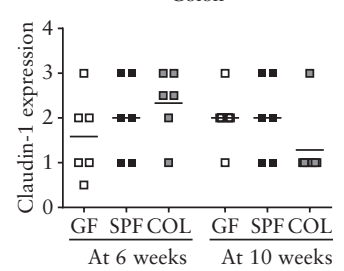

$\mathrm{E}$

GF

$\mathrm{SPF}$
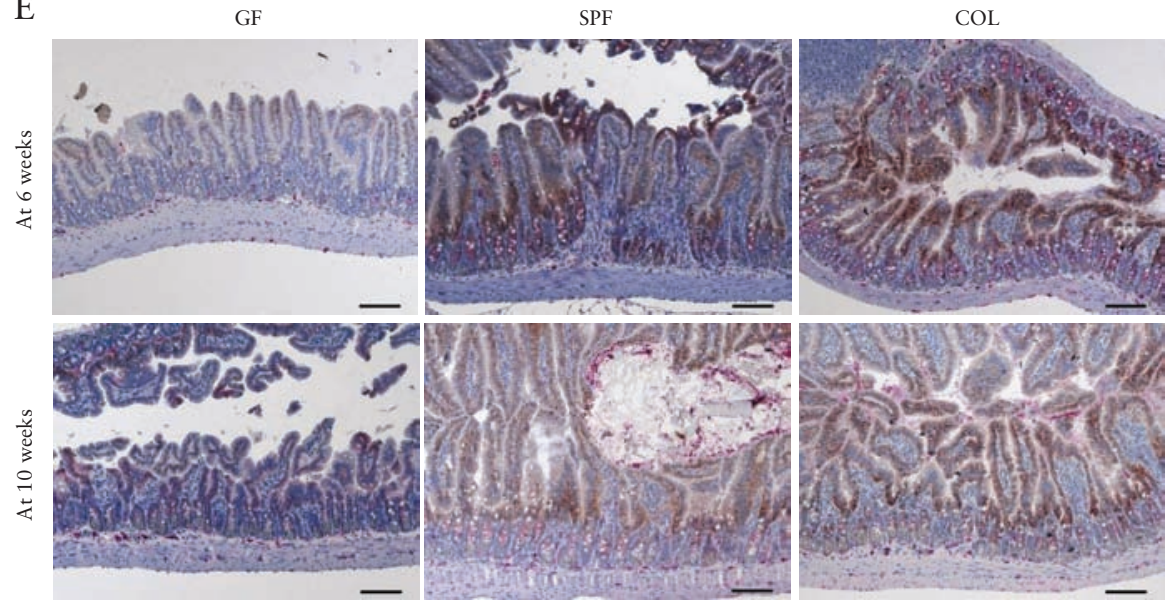

Figure 1. The luminal microbiota affects intestinal microarchitecture. [A] Germ-free [GF] mice were colonized [COL] by oral gavage at the age of 5 weeks with specific-pathogen-free [SPF] microbiota. COL mice were compared with GF and SPF mice at the age of 6 and 10 weeks. [B]The thickness of the ileum and colon mucosa was measured from histological sections. [C] Representative sections of the ileum stained with haematoxylin/eosin are shown. [D] Claudin 1 expression determined in sections stained immunohistologically. [E] Representative sections of the ileum immunohistologically stained for claudin 1 [brown] and CD163 [red]. Counterstain was performed with haematoxylin. Scale bar, $100 \mu \mathrm{m}$. Data are from 2-8 mice, of which at least two sites of the mucosa were measured [B]. Data are from 6-7 mice from at least two experiments [D]. Two-way ANOVA with the Holm-Sidak test in the respective group ${ }^{*} p<0.05,{ }^{* *} p<0.01,{ }^{* * *} p<0.001$.

of $\mathrm{MHCII}^{+}$macrophages was detectable in COL mice 1 week after colonization in comparison with age-matched GF and SPF mice [Figure 3B].
An RNA-sequencing analysis of ileum tissue revealed differences in selected macrophage-associated genes between GF and SPF mice [Figure 3C] at 6 and 10 weeks of age. Among them, Msr-1, Itgax, 
A
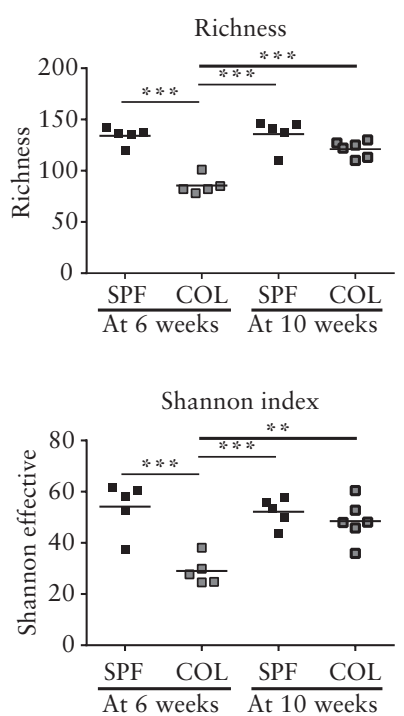

D

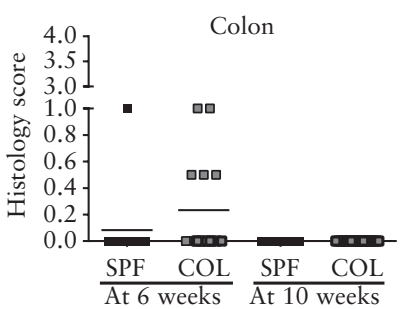

B

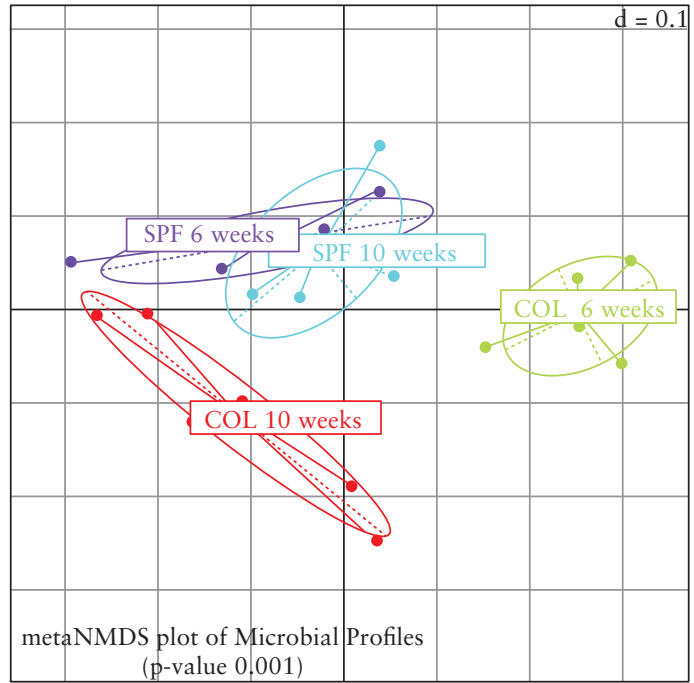

C

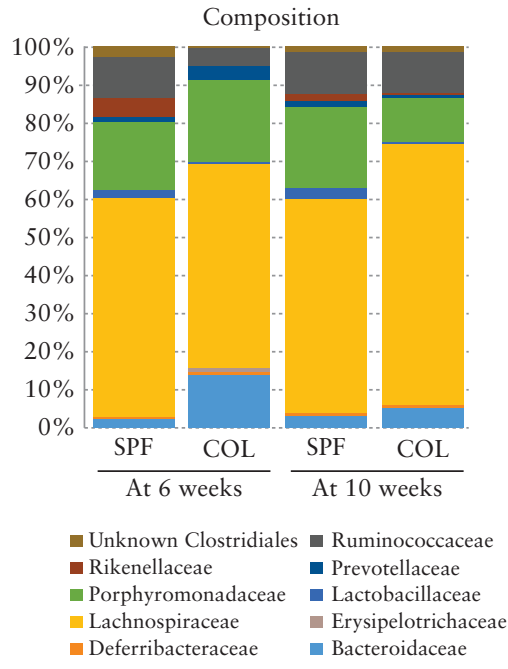

Figure 2. Colonization in Week 5 suffices to induce inflammation. Germ-free [GF] mice were colonized [COL] orally by gavage at the age of 5 weeks with specificpathogen-free [SPF] microbiota. COL mice were compared with SPF mice at the age of 6 and 10 weeks. [A] Richness and Shannon effective species counts in caecal content are demonstrated. [B] Non-metrical multiple-dimensional scaling analysis [ $\beta$-diversity] showing separation of caecal bacterial communities in SPF and COL mice. [C] Caecal bacterial composition [relative abundance of total sequences] at the family level is shown. [D]The histological scores of the colon from SPF and COL mice at Week 6 and Week 10 are presented. [E] Representative sections from colon stained with haematoxylin/eosin are shown. Scale bar, $20 \mu \mathrm{m} . n=6-23$ from at least two experiments and the mean are shown. Two-way ANOVA with Holm-Sidak test, ${ }^{*} p<0.05,{ }^{* *} p<0.01,{ }^{* * *} p<0.001[\mathrm{~A}]$.

Itgae, Ly6c2, Cx3cr1, and Nos2 were significantly $\left[p_{\text {adj }}<0.1\right]$ upregulated in SPF mice at 10 weeks. When SPF and COL mice were compared, regarding macrophage-related genes only, $\operatorname{Ly} 6 c 2\left[p_{\text {adj }}<0.1\right]$ was significantly upregulated in 6-week-old COL mice. No significant differences in expression of the selected genes were found between SPF and COL mice 5 weeks after colonization [Figure 3C].

A gene-set enrichment analysis with gene ontology [GO] gene sets confirmed our analysis with selected markers [Supplementary Figure $3 \mathrm{~A} / \mathrm{B}]$. Expression analysis of core-enriched genes of selected highly enriched GO gene sets related to macrophage biology showed clear differential expression patterns in the ileum of GF compared with in the ileum of SPF mice at either age. The expression pattern of COL mice 1 week after colonization did show more similarity to that of GF mice than to that of SPF mice. Five weeks after colonization,
COL mice equalled SPF mice with respect to the expression pattern of the selected GO gene sets [Supplementary Figure 3C].

In health, LPMCs from SPF mice are known to be unresponsive to LPS stimulation. ${ }^{30}$ In line with this, LPS stimulation of LPMCs isolated from the colon of SPF or COL mice did not result in an increased TNF $\alpha$ production. Only in macrophages from the colon of GF mice could LPS-induced TNF $\alpha$ expression be detected [Figure 3D]. These results suggest a desensitization mediated by the colonization of COL mice. However, LPS stimulation of ileal LPMCs from GF and SPF mice resulted in an increased proportion of TNF $\alpha$ expressing macrophages compared with similar treatment of LPMCs from COL mice after 5 weeks of colonization [Figure 3D].

The results of the RNA expression analysis together with the flow cytometry results provided more in-depth evidence that 5 weeks 
A
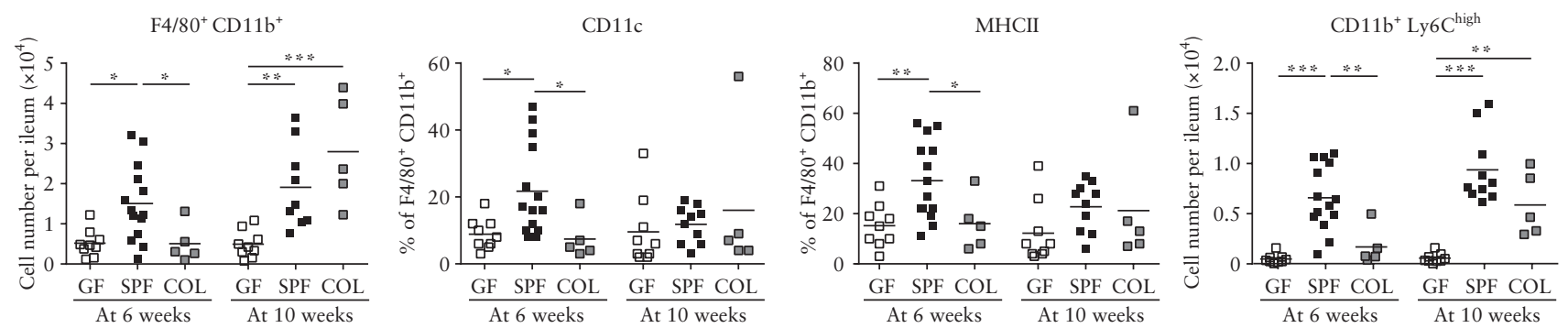

B
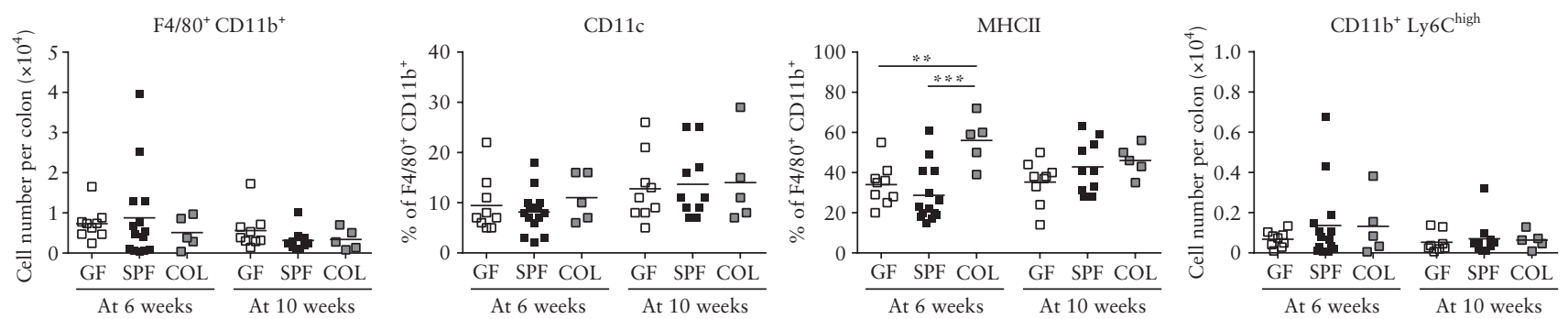

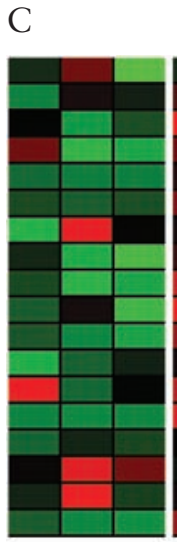

GF

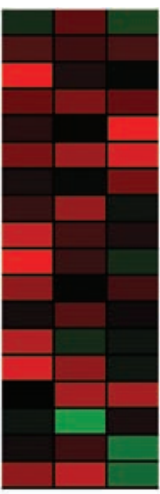

SPF At 6 weeks

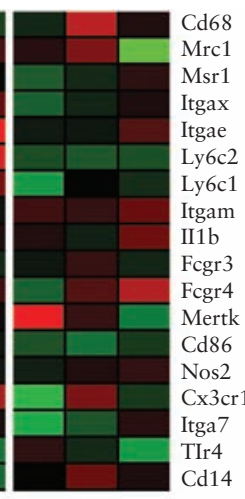

COL

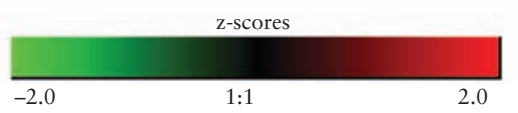

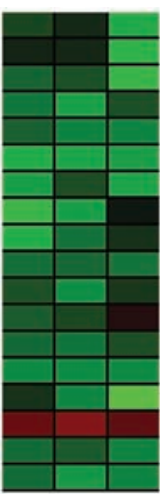

GF

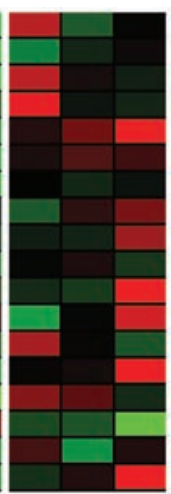

SPF

At 10 weeks

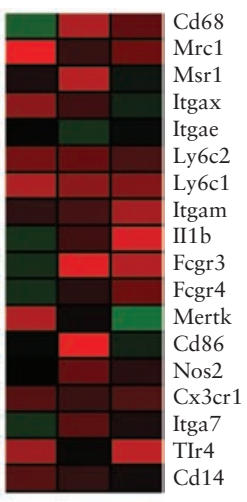

COL

Figure 3. Microbiota causes a time-dependent restoration of the myeloid compartment in the ileum. The intestines of germ-free [GF], specific-pathogen-free [SPF] mice, and GF mice colonized at the age of 5 weeks [COL] were analysed at the age of 6 and 10 weeks. Lamina propria mononuclear cells [LPMCs] were isolated from the distal $10 \mathrm{~cm}$ of small intestine [ileum] or the whole colon. The myeloid marker expression of LPMCs isolated from [A] ileum or [B] colon was assessed by flow cytometry. [C] Heatmap of selected macrophage marker expression analysed in total RNA sequencing of whole ileum tissue. $z$-scores are given. [D] LPMCs were stimulated with $1 \mu \mathrm{g} / \mathrm{mL}$ lipopolysaccharide [LPS] as described in the Methods. TNF $\alpha$ producing F4/80 ${ }^{+} \mathrm{CD}_{11} \mathrm{~b}^{+}$macrophages of $10-$ weekold mice are shown. $n=9-10$ from at least two experiments and the mean are shown. Two-way ANOVA with Holm-Sidak test, ${ }^{*} p<0.05,{ }^{* *} p<0.01, * * * p<0.001$. 
after colonization, adaption of the macrophage compartment had been completed.

Thus, a restoration and adaption of the macrophage compartment following colonization did occur in a time-dependent manner.

\subsection{The microbiota was necessary for the infiltration of macrophages after DSS treatment}

The increased susceptibility of GF mice to DSS is well described. ${ }^{56-58}$ By performing ex vivo Ussing chamber experiments comparing GF and SPF mice, we aimed to further specify this barrier defect in health. The colon of GF mice revealed a lower total electrical resistance compared with that of SPF mice, and this was due to a decreased subepithelial resistance [Figure 4A]. The paracellular [mannitol] flux was increased in GF mice [Figure 4B], whereas the transcytotic transport of horseradish peroxidase was not influenced by the presence or absence of microbiota [Figure 4C]. Thus the barrier defect can be predominantly attributed to a subepithelial defect.

To analyse the effect of microbiota functionally, DSS-induced colitis was induced, as a barrier-disrupting model [Figure 4D]. As is already known for other mouse strains, ${ }^{56,57}$ wild-type GF mice are more susceptible to DSS compared with SPF mice, and die at rather low concentrations of DSS [Supplementary Figure 4A]. Thus, in all further experiments a DSS concentration of $1.5 \%$ was applied. To analyse the macrophage compartment, the experiment was stopped at Day 5, and clinical signs of colitis were analysed. Clinical and histologic colitis scores did not differ between GF and SPF mice after DSS exposure [Supplementary Figure 4B/C]. Colon culture revealed an increased production of IFN $\gamma$ and TNF $\alpha$ in SPF mice, but not in GF mice, exposed to DSS [Supplementary Figure 4D]. Accordingly, a significant increase in epithelial apoptosis was observed only in GF mice upon DSS exposure [Supplementary Figure 4E].

An increase in the frequency of recently infiltrated monocytes and macrophages was only seen in SPF mice exposed to DSS, but not in GF mice exposed to DSS [Figure 4E]. From these findings, we conclude that the presence of the luminal microbiota is mandatory for inducing infiltration of macrophages that ultimately mediate inflammation. The expression of claudin 1 did not change upon DSS treatment in GF or SPF mice [Supplementary Figure 4H]. In addition, it is not inflammation, but rather the pure barrier defect, that explains the increased susceptibility of GF mice to DSS.

\subsection{DSS treatment induced infiltration of macrophages independent from the time point of colonization}

DSS treatment was also applied in SPF and COL mice late after colonization to assess whether the time point of colonization had functional differences [Figure 5A]. In contrast to GF mice, colonization with SPF microbiota rescued these mice from dying of DSS exposure
A

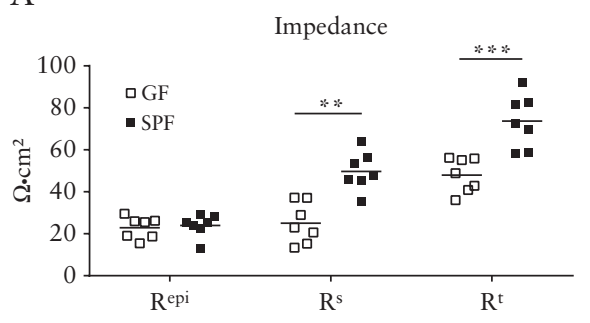

B

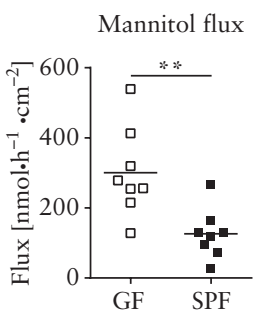

C

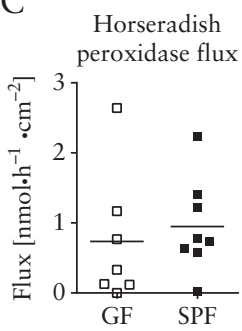

E

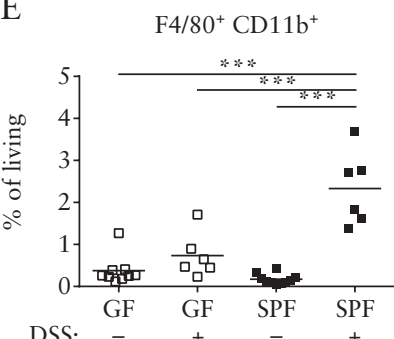

$\mathrm{D}$

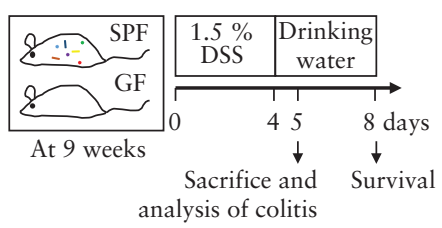

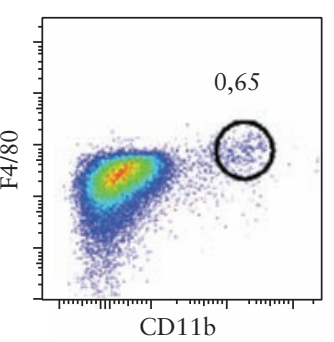

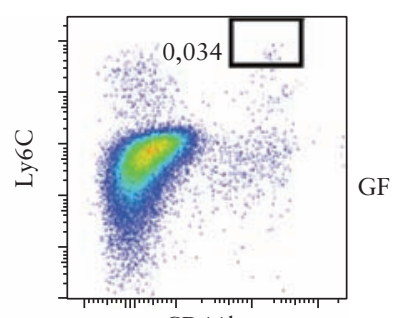

CD11b

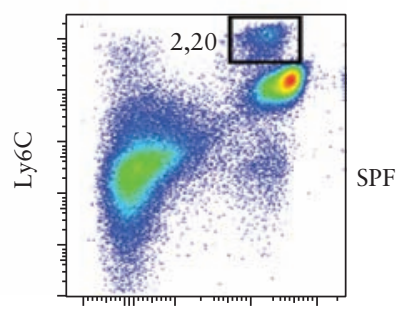

CD11b

Figure 4. Functional impact of bacterial colonization. Analysis of barrier function was performed in Ussing chamber experiments. [A] Impedance measurements displaying epithelial $\left[R^{\text {epi }}\right]$, subepithelial $\left[R^{s}\right]$, and total resistance $\left[R^{t}\right]$ of colon from GF and SPF mice. [B] Mannitol and [C] horseradish peroxidase flux of colon tissue were determined in Ussing chamber experiments. [D] DSS treatment of GF and SPF mice was performed for 4 days followed by 1 day of normal drinking water. [E] The lamina propria mononuclear cells were isolated from the colon. The expression of myeloid marker was assessed by flow cytometry. Representative Dot plots of living cells are presented. $n=7-8$ from four experiments and the mean are shown, Mann-Whitney $U$ test $* * p<0.01,{ }^{* * *} p<0.001$ [A-C]. $n=6-10$ from two experiments and the mean are shown. Two-way ANOVA with Holm-Sidak test, ${ }^{*} p<0.05,{ }^{* *} p<0.01,{ }^{* * *} p<0.001$ [E]. 
[Figure 5B]. As indicated by survival, SPF and COL mice presented with comparable colitis severity [Figure 5B]. Remarkably, histologic inflammation was more pronounced in DSS-exposed SPF than in DSS-exposed COL mice [Figure 5C]. Claudin 1 expression did not change after DSS treatment [Figure 5D]. Immunohistological detection of F4/80 showed that the quantitative number of macrophages increased in SPF and COL mice treated with DSS [Figure 5E]. Flow cytometric analyses of colonic macrophages revealed an infiltration into the lamina propria in both DSS-treated groups. The frequency of CD11c and MHCII did not change in DSS-treated mice compared with the frequencies in control mice [Figure 5F]. An RNA sequencing analysis of colon tissue revealed no significant differences regarding selected macrophage-related genes between SPF and COL mice. Interestingly, after colitis induction in SPF mice, Itgax, Ly6c1, Itgam, and Nos 2 were significantly $\left[p_{\text {adi }}<0.1\right]$ upregulated compared with in the untreated SPF mice. In COL mice treated with DSS, Ly6C1,
A

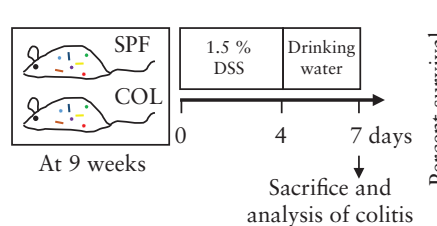

$\mathrm{E}$

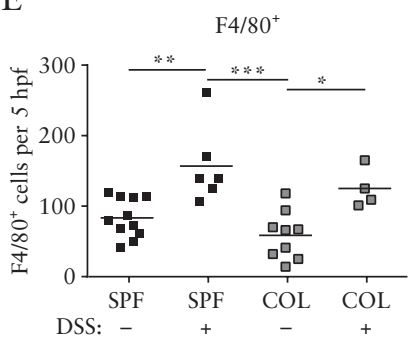

B

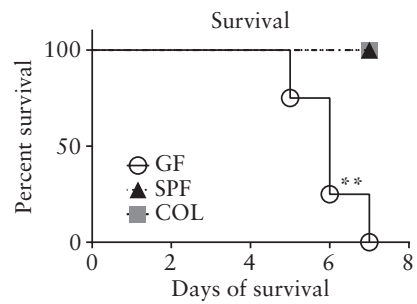

$\mathrm{F}$

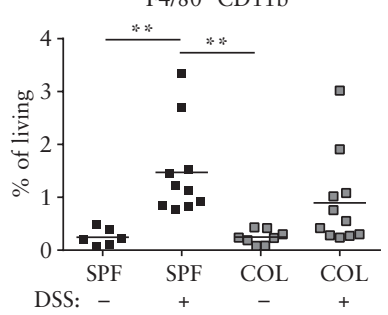

C

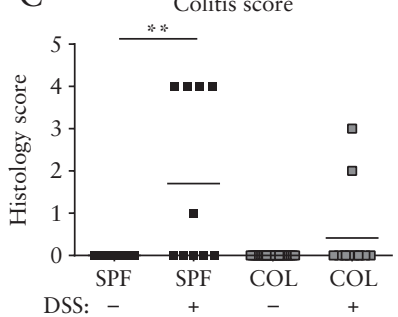

D

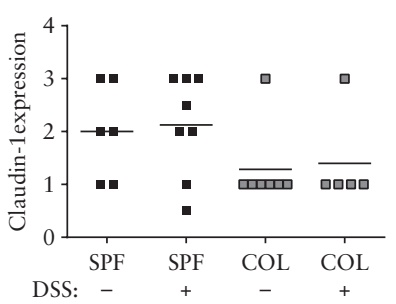

CD11c

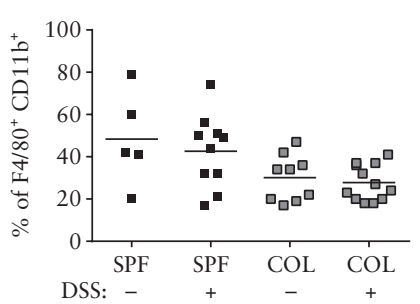

MHCII

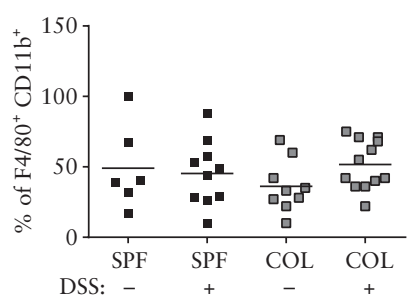

G

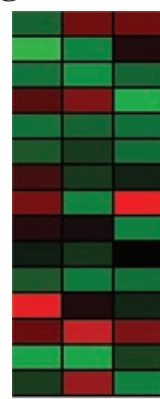

SPF

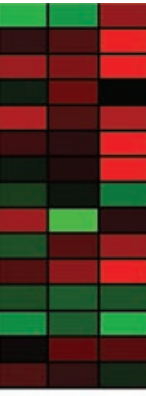

SPF

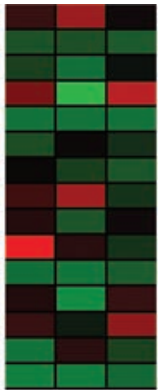

COL

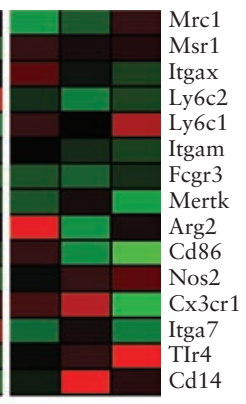

$\mathrm{H}$
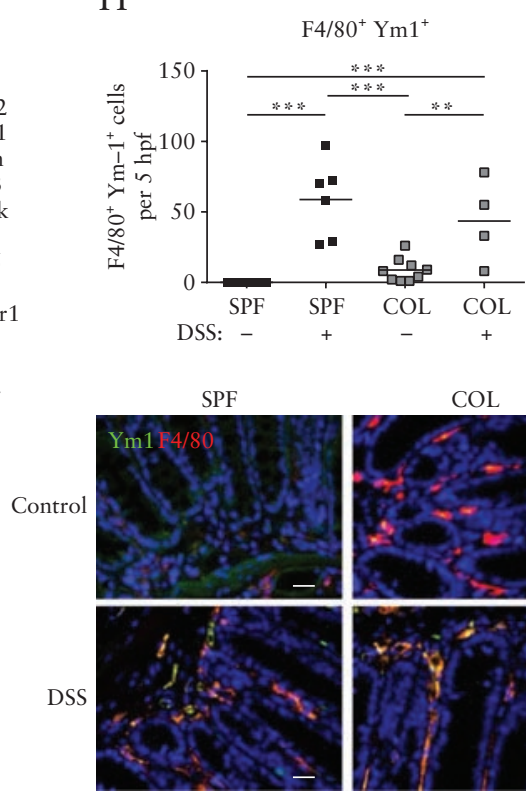

Figure 5. The time point of colonization is irrelevant for the composition of the myeloid compartment following DSS exposure. [A]The experimental set-up of DSS-induced colitis is illustrated. [B] The survival curve throughout the experiment is shown. [C] The histological inflammation score of the colon after DSS treatment of SPF mice and GF mice colonized at the age of 5 weeks [COL] is presented. [D] Immunohistological assessment of claudin 1 expression on colon sections. Claudin 1 expression was determined using a score described in detail in the Methods section. [E] Immunohistological staining of colon sections for $\mathrm{F} 4 / 80^{+}$macrophages. Positive cells were counted in histological sections per five high-power fields $\left[1 \mathrm{hpf}^{2} 0.237 \mathrm{~mm}^{2}\right]$. [F] The myeloid marker expression of lamina propria mononuclear cells of the colon was assessed by flow cytometry. [G] Heatmap of selected macrophage marker expression analysed in total RNA sequencing of whole ileum tissue. z-scores are given. [H] Immunofluorescence staining of colon sections for Ym1 [Alexa488, green] and F4/80 [FastRed, red]. Nuclei were stained with DAPI [blue]. Positive cells were counted in histological sections per five high-power fields. Scale bar, $20 \mu \mathrm{m}$. $n=5-12$ from at least two experiments and the mean are shown. Log-Rank [Cox-Mantel] test versus 3.5\% DSS, ${ }^{* *} p<0.01$ [B]. Two-way ANOVA with Holm-Sidak test, ${ }^{*} p<0.05,{ }^{* *} p<0.01$, ${ }^{* * *} p<0.001[\mathrm{D}, \mathrm{E}, \mathrm{F}, \mathrm{H}]$. Mann-Whitney U test ${ }^{*} p<0.05,{ }^{* *} p<0.01,{ }^{* *} p<0.001[\mathrm{C}]$. 
Nos2, and $C d 14$ showed a significant $\left[p_{\text {adj }}<0.1\right]$ increase in expression [Figure 5G]. The number of macrophages expressing Ym1 was significantly increased in DSS-TReated mice were compared to health non-DSS exposed animals [Figure $5 \mathrm{H}]$.

These data indicated that colonization with microbiota was a prerequisite not only for inflammation but also for macrophage infiltration. However, the time point of colonization in life did not play a critical role in macrophage development. Nevertheless, the restoration of macrophages required longer than the structural adaptation after microbial colonization.

\section{Discussion}

The intestinal homeostasis in the lamina propria is regulated by the intestinal microbiota. ${ }^{3-6}$ Previous data analysing NKT cell function indicate that a delayed intestinal colonization of GF mice drives the pro-inflammatory capacity of this cell population. ${ }^{13}$ This relates to the concept of the hygiene hypothesis, in which exposure to antigens early in life has been considered to protect from diseases such as IBD later in life. ${ }^{18}$ With the present study, we broaden this view by defining the impact of the luminal microbiota on the development and function of intestinal macrophages as well as on mucosal integrity.

Previous work indicates that the myeloid compartment can adapt to the local milieu. This has been demonstrated for monocytes that differentiate in the inflamed colon to pro-inflammatory myeloid cells. ${ }^{38,39}$ Thus, targeting this plasticity either from the mucosal or from the luminal site might open novel therapeutic strategies for IBD. This concept has indirectly been proven. A study by Vos et al. showed that after anti-TNF $\alpha$ treatment, anti-inflammatory macrophages are induced, whereas in untreated Crohn's disease patients, lamina propria pro-inflammatory macrophages are predominant. ${ }^{26}$

In line with this, work from our group indicates that the mucosa of patients with Crohn's disease is densely infiltrated with proinflammatory macrophages, whereas the adjacent mesenteric fat tissue is predominantly infiltrated by rather anti-inflammatory macrophages. ${ }^{25,59}$ This ability of myeloid cells to adapt to the local milieu suggests that the mucosal macrophage compartment may be able to develop, even when intestinal colonization occurs later in life. To address this question, GF mice were colonized at Week 5, and the macrophage compartment was analysed at Week 6 and Week 10. In the ileum, a time-dependent restoration of macrophages and recently infiltrated monocytes occurred. One week after colonization, the number of macrophages equalled the number in GF mice. However, the macrophage compartment adapted numerically 5 weeks after the colonization to the number of macrophages in SPF mice, which were colonized since birth. RNA analysis confirmed differences regarding macrophage-related genes in GF mice compared with those in SPF mice. The expression of macrophage-related genes in COL and SPF mice 5 weeks after colonization was similar. Interestingly, COL mice at Week 6 showed a significant upregulation of $L y 6 c 2$, indicating the microbiota-driven influx of monocytes into the ileum, even though the number of monocytes was equal to that of GF mice at this time point.

Interestingly, one study showed that in broad-spectrum antibiotic-treated mice recolonized with SPF microbiota $\mathrm{F} 4 / 80^{+}$macrophages and monocytes in the ileum have already recovered 7 days after the faecal microbiota transfer. ${ }^{60}$ From this, we conclude that priming of the immune system before colonization influences the immunological response and restoration of immune cells in the gut. Furthermore, one has to consider the time point of colonization, since the time point of the first contact with distinct microbiota determines the immunological reactions later on. ${ }^{61} \mathrm{~A}$ colonization in the pre-weaning phase could result in different findings, since macrophages in this phase of life are mostly embryo-derived. ${ }^{33}$ Future studies will have to define the 'window of opportunity' and thus the time point of colonization, with a particular focus on earlier time points.

In line with previous data, we did not identify a microbiota-dependent effect for the colonic macrophage compartment. However, this study did not focus on time-dependent adaption of this compartment after colonization..$^{38}$ Conversely, another study reported a reduced number of colonic macrophages in GF C57BL/6 mice. Notably, previous studies have suggested that other intestinal immune cells are developing in a microbiota-dependent manner. For instance, the commensal microbiota has been shown to be required for the expansion of pro-inflammatory $\mathrm{CD}^{+} \mathrm{T}$ cells in the colon ${ }^{6}$ and for the regulation of innate lymphoid cells in the small intestine. ${ }^{62}$ Some studies have suggested that there is a critical window of opportunity for the first contact with microbiota. ${ }^{63}$ Olszak et al. revealed that the time point of colonization after birth affects the composition of the natural killer T [NKT] cell compartment, inducing a more pro-inflammatory state when colonization occurs later in life. ${ }^{13}$ Furthermore, humoral immunity is also regulated by microbial colonization during a critical time early in life. At this time-point, serum IgE levels are modulated and ensure a functional immune regulation throughout life. ${ }^{15}$

Lipopolysaccharide stimulation of colonic macrophages from GF mice resulted in the expression of TNF $\alpha$. In contrast, colonic macrophages from SPF mice were unresponsive to LPS, which is in line with previous data indicating that colonic macrophages are hyporeactive to Toll-like-receptor ligands. ${ }^{30}$ The authors of that study demonstrated that the microbiota induces IL-10 expression in the colon and thus inhibits inflammatory responses of macrophages. ${ }^{30}$ Consistent with that, and supporting the functional restoration of the macrophage compartment, macrophages isolated from COL mice at Week $10 \mathrm{did}$ not respond to LPS stimulation. These functional analyses indicate that, although no quantitative and phenotypic differences were detectable, fully functional colonic macrophages require the presence of intestinal microbiota. Remarkably, the ileal macrophages compartment differed from that of the colon. In our study, the expression of TNF $\alpha$ in the ileum after LPS stimulation was higher in SPF than in COL mice. Thus, immunological differences between these two intestinal segments ${ }^{64}$ will have to be defined in more detail in future studies.

Having established the fact that the correct luminal microbiota is a prerequisite for the infiltration of monocytes into the lamina propria, we further wanted to define functional properties under inflammatory conditions. For this, an additional barrier defect was induced by oral DSS administration. In accordance with our hypothesis that intestinal antigens are required for monocytes to infiltrate the intestinal mucosa, an increase in this cell population was only seen in SPF but not in GF mice upon DSS exposure. Hence, it is not surprising that neither previous work nor our data detected signs of inflammation in the DSS-exposed GF mice, as indicated by the lack of upregulation of pro-inflammatory cytokines and the lack of cell infiltration. ${ }^{56,65}$ The same result was obtained in an ileitis mouse model using TNF ${ }^{\text {deltaARE }}$ mice. ${ }^{66,67}$

While it is known that GF mice are more susceptible to DSS, the mechanism behind this has only partially been resolved. In our previous data, by applying a barrier model system, pro-inflammatory macrophages induced a significant decrease in epithelial resistance, 


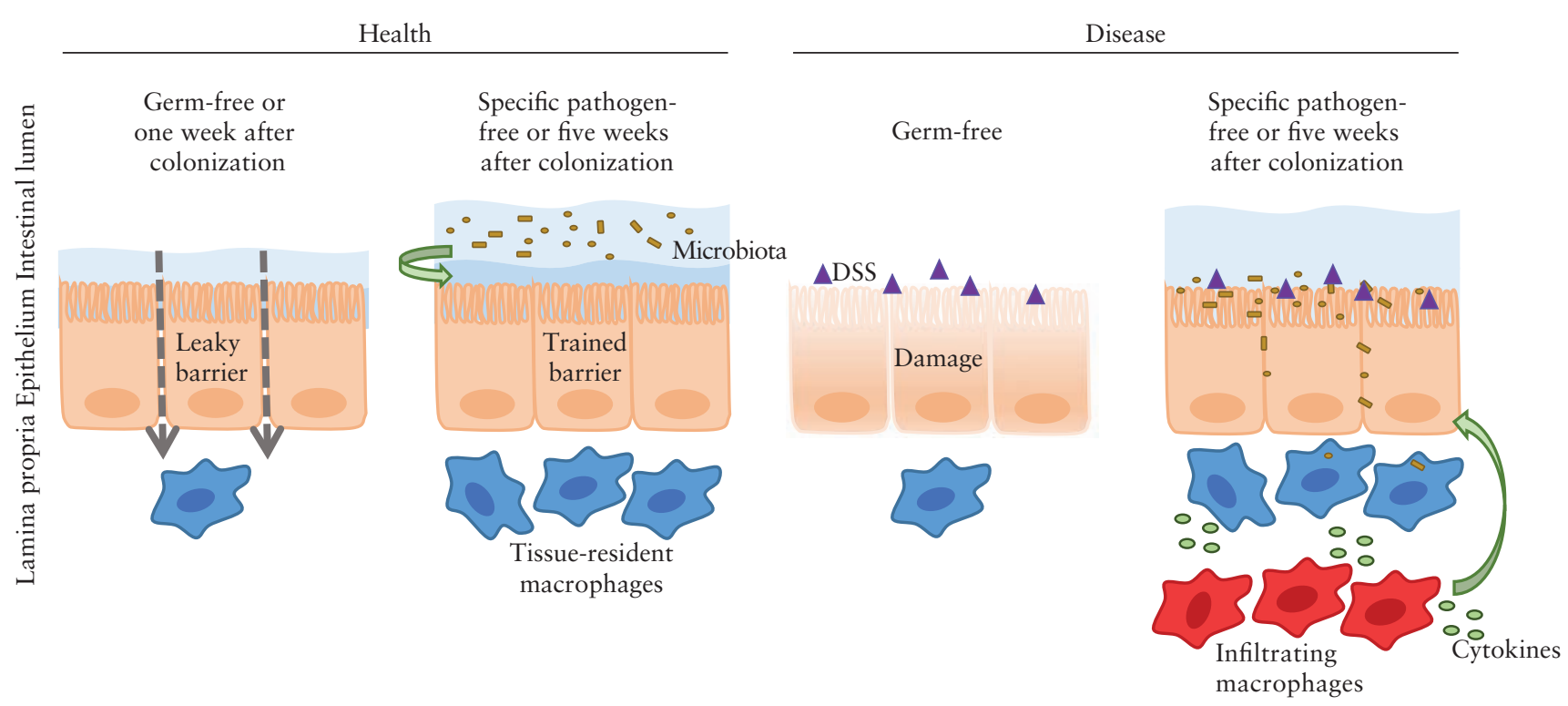

Figure 6. Proposed model. When comparing germ-free [GF] mice versus specific-pathogen-free [SPF] mice, the intestinal barrier function presents with a striking difference in several aspects. The gut of both GF mice and mice that have only shortly been exposed to microbiota reveal a barrier leakage with a profoundly reduced number of [ileum] or dysfunctional [colon] macrophages. Colonization from birth or later in life, with an adaption period of several weeks, allows for restoration of the intestinal macrophages compartment. These are prerequisites for resisting stressors from the luminal site. In our experiments, mice colonized with microbiota [either at birth or later in life] were partly protected from DSS treatment, whereas GF mice succumbed to death. The formation of a functional mucosal macrophage compartment is dependent on the intestinal microbiota.

whereas anti-inflammatory macrophages were barrier protective. ${ }^{25}$ As expected, the electrical resistance and mannitol flux measurements revealed a barrier leakage in GF mice in the present study. Thus, it can be suggested that macrophage dysfunction in the absence of luminal microbiota might contribute to barrier leakage. Recent data indicate that downregulation of tight junction proteins, and hence a structural epithelial barrier defect, mediates this high susceptibility. ${ }^{56,57}$ Additional data indicate that Toll-like receptor signalling in epithelial cells is crucial for gut injury and associated mortality. ${ }^{68}$ Intestinal microbiota, as well as Myd88 signalling, has been shown to exert a profound impact on the regeneration capacity of the epithelium. ${ }^{69}$

Since we observed a restoration of the colonic macrophage compartment several weeks after colonization, we used these mice to test for functional differences by the use of DSS. Although we did not identify a difference between the macrophage compartment of SPF and COL mice, DSS exposure in SPF mice resulted in a higher histologic colitis score when compared with COL mice. The finding of mild intestinal inflammation 1 week after colonization suggests a desensitization that mediates the decreased inflammatory response in the DSS-induced colitis model.

Our data reemphasize the necessity of microbial colonization for a functional intestinal barrier [Figure 6]. As previously indicated for NKT cells and innate lymphoid cells, intestinal colonization forms a prerequisite for the infiltration and development of the ileal and colonic macrophage compartment, and hence for intestinal homeostasis. However, infiltrating monocytes maintain their plasticity and hence can develop this compartment at any time point in life; however, they require several weeks of adaptation. Thus, targeting this plasticity, either from the mucosal or from the luminal site, might open novel therapeutic strategies for reducing intestinal inflammation.

\section{Funding}

This work was supported by the Deutsche Forschungsgemeinschaft [DFG] [SI 749/7-1 to BS and MB; SI 749/10-1 to BS and TRR 241 to BS and ZT].

\section{Conflict of interest}

The authors declare that the research was conducted in the absence of any commercial or financial relationships that could be construed as a potential conflict of interest.

\section{Acknowledgments}

We thank Inka Freise, Simone Spieckermann, Sabine Schmidt, and Anja Fromm for technical assistance. We are grateful to Carl Weidinger for critical reading and discussion. The results are part of the PhD thesis of FS.

\section{Author Contributions}

FS, KD, AB, GL, JK, HW, MF, MiS, AK, CR, and RG planned and performed the experiments and the associated analysis. KD and MoS analysed the microbiota data. HH and ZT analysed the RNA sequencing data. MB and BS initiated the project and the experimental concept. FS and BS drafted and wrote the manuscript.

\section{Supplementary Data}

Supplementary data are available at ECCO-JCC online.

\section{References}

1. Molodecky NA, Soon IS, Rabi DM, et al. Increasing incidence and prevalence of the inflammatory bowel diseases with time, based on systematic review. Gastroenterology 2012;142:46-54.e42; quiz e30. 
2. Franke A, McGovern DP, Barrett JC, et al. Genome-wide meta-analysis increases to 71 the number of confirmed Crohn's disease susceptibility loci. Nat Genet 2010;42:1118-25.

3. Ohnmacht C, Park JH, Cording S, et al. MUCOSAL IMMUNOLOGY. The microbiota regulates type 2 immunity through ROR $\gamma t^{+} \mathrm{T}$ cells. Science 2015;349:989-93.

4. Mortha A, Chudnovskiy A, Hashimoto D, et al. Microbiota-dependent crosstalk between macrophages and ILC3 promotes intestinal homeostasis. Science 2014;343:1249288.

5. Niess JH, Adler G. Enteric flora expands gut lamina propria CX3CR1+ dendritic cells supporting inflammatory immune responses under normal and inflammatory conditions. J Immunol 2010;184:2026-37.

6. Niess JH, Leithäuser F, Adler G, Reimann J. Commensal gut flora drives the expansion of proinflammatory CD4 $\mathrm{T}$ cells in the colonic lamina propria under normal and inflammatory conditions. J Immunol 2008;180:559-68.

7. Hooper LV, Wong MH, Thelin A, Hansson L, Falk PG, Gordon JI. Molecular analysis of commensal host-microbial relationships in the intestine. Science 2001;291:881-4.

8. Stappenbeck TS, Hooper LV, Gordon JI. Developmental regulation of intestinal angiogenesis by indigenous microbes via Paneth cellsProc Natl Acad Sci U S A 2002;99:15451-5.

9. Riedler J, Braun-Fahrländer C, Eder W, et al.; ALEX Study Team. Exposure to farming in early life and development of asthma and allergy: a cross-sectional survey. Lancet 2001;358:1129-33.

10. Schuijs MJ, Willart MA, Vergote K, et al. Farm dust and endotoxin protect against allergy through A20 induction in lung epithelial cells. Science 2015;349:1106-10.

11. Hviid A, Svanström H, Frisch M. Antibiotic use and inflammatory bowel diseases in childhood. Gut 2011;60:49-54.

12. Shaw SY, Blanchard JF, Bernstein CN. Association between the use of antibiotics in the first year of life and pediatric inflammatory bowel disease. Am J Gastroenterol 2010;105:2687-92.

13. Olszak T, An D, Zeissig S, et al. Microbial exposure during early life has persistent effects on natural killer T cell function. Science 2012;336:489-93.

14. Herbst T, Sichelstiel A, Schär C, et al. Dysregulation of allergic airway inflammation in the absence of microbial colonization. Am J Respir Crit Care Med 2011;184:198-205.

15. Cahenzli J, Köller Y, Wyss M, Geuking MB, McCoy KD. Intestinal microbial diversity during early-life colonization shapes long-term IgE levels. Cell Host Microbe 2013;14:559-70.

16. Hill DA, Siracusa MC, Abt MC, et al. Commensal bacteria-derived signals regulate basophil hematopoiesis and allergic inflammation. Nat Med 2012;18:538-46.

17. McCoy KD, Harris NL, Diener P, et al. Natural IgE production in the absence of MHC Class II cognate help. Immunity 2006;24:329-39.

18. Okada H, Kuhn C, Feillet H, Bach JF. The 'hygiene hypothesis' for autoimmune and allergic diseases: an update. Clin Exp Immunol 2010;160:1-9.

19. Smith PD, Smythies LE, Mosteller-Barnum M, et al. Intestinal macrophages lack CD14 and CD89 and consequently are down-regulated for LPS- and IgA-mediated activities. J Immunol 2001;167:2651-6.

20. Smythies LE, Shen R, Bimczok D, et al. Inflammation anergy in human intestinal macrophages is due to Smad-induced IкB $\alpha$ expression and NF-кB inactivation. J Biol Chem 2010;285:19593-604.

21. Adib-Conquy M, Cavaillon J-M. Gamma interferon and granulocyte/ monocyte colony-stimulating factor prevent endotoxin tolerance in human monocytes by promoting interleukin- 1 receptor-associated kinase expression and its association to MyD88 and not by modulating TLR4 expression. J Biol Chem 2002;277:27927-34.

22. Medvedev AE, Lentschat A, Wahl LM, Golenbock DT, Vogel SN. Dysregulation of LPS-induced Toll-like receptor 4-MyD88 complex formation and IL-1 receptor-associated kinase 1 activation in endotoxintolerant cells. J Immunol 2002;169:5209-16.

23. Rugtveit J, Bakka A, Brandtzaeg P. Differential distribution of B7.1 [CD80] and B7.2 [CD86] costimulatory molecules on mucosal macrophage subsets in human inflammatory bowel disease [IBD]. Clin Exp Immunol 1997;110:104-13.
24. Chang PV, Hao L, Offermanns S, Medzhitov R. The microbial metabolite butyrate regulates intestinal macrophage function via histone deacetylase inhibition. Proc Natl Acad Sci U S A 2014;111:2247-52.

25. Lissner D, Schumann M, Batra A, et al. Monocyte and M1 macrophageinduced barrier defect contributes to chronic intestinal inflammation in IBD. Inflamm Bowel Dis 2015;21:1297-305.

26. Vos AC, Wildenberg ME, Duijvestein M, Verhaar AP, van den Brink GR, Hommes DW. Anti-tumor necrosis factor- $\alpha$ antibodies induce regulatory macrophages in an Fc region-dependent manner. Gastroenterology 2011;140:221-30

27. Vos AC, Wildenberg ME, Arijs I, et al. Regulatory macrophages induced by infliximab are involved in healing in vivo and in vitro. Inflamm Bowel Dis 2012;18:401-8.

28. Caprioli F, Bosè F, Rossi RL, et al. Reduction of CD68 $8^{+}$macrophages and decreased IL-17 expression in intestinal mucosa of patients with inflammatory bowel disease strongly correlate with endoscopic response and mucosal healing following infliximab therapy. Inflamm Bowel Dis 2013;19:729-39.

29. Steinbach EC, Plevy SE. The role of macrophages and dendritic cells in the initiation of inflammation in IBD. Inflamm Bowel Dis 2014;20:166-75.

30. Ueda Y, Kayama H, Jeon SG, et al. Commensal microbiota induce LPS hyporesponsiveness in colonic macrophages via the production of IL-10. Int Immunol 2010;22:953-62.

31. Mass E, Ballesteros I, Farlik M, et al. Specification of tissue-resident macrophages during organogenesis. Science 2016;353:aaf4238.

32. Schulz C, Gomez Perdiguero E, Chorro L, et al. A lineage of myeloid cells independent of Myb and hematopoietic stem cells. Science 2012;336:86-90.

33. Bain CC, Bravo-Blas A, Scott CL, et al. Constant replenishment from circulating monocytes maintains the macrophage pool in the intestine of adult mice. Nat Immunol 2014;15:929-37.

34. De Schepper S, Verheijden S, Aguilera-Lizarraga J, et al. Self-maintaining gut macrophages are essential for intestinal homeostasis. Cell 2018;175:400-15.e13.

35. Shaw TN, Houston SA, Wemyss K, et al. Tissue-resident macrophages in the intestine are long lived and defined by Tim- 4 and CD4 expression. J Exp Med 2018;215:1507-18.

36. Quévrain E, Maubert MA, Michon C, et al. Identification of an anti-inflammatory protein from Faecalibacterium prausnitzii, a commensal bacterium deficient in Crohn's disease. Gut 2016;65:415-25.

37. Zigmond E, Bernshtein B, Friedlander G, et al. Macrophage-restricted interleukin-10 receptor deficiency, but not IL-10 deficiency, causes severe spontaneous colitis. Immunity 2014;40:720-33.

38. Rivollier A, He J, Kole A, Valatas V, Kelsall BL. Inflammation switches the differentiation program of Ly6Chi monocytes from antiinflammatory macrophages to inflammatory dendritic cells in the colon. J Exp Med 2012;209:139-55.

39. Zigmond E, Varol C, Farache J, et al. Ly6C hi monocytes in the inflamed colon give rise to proinflammatory effector cells and migratory antigenpresenting cells. Immunity 2012;37:1076-90.

40. Wesolowska-Andersen A, Bahl MI, Carvalho V, et al. Choice of bacterial DNA extraction method from fecal material influences community structure as evaluated by metagenomic analysis. Microbiome 2014;2:19.

41. Klindworth A, Pruesse E, Schweer T, et al. Evaluation of general $16 \mathrm{~S}$ ribosomal RNA gene PCR primers for classical and next-generation sequencing-based diversity studies. Nucleic Acids Res 2013;41:e1.

42. Lagkouvardos I, Joseph D, Kapfhammer M, et al. IMNGS: A comprehensive open resource of processed $16 \mathrm{~S}$ rRNA microbial profiles for ecology and diversity studies. Sci Rep 2016;6:33721.

43. Lagkouvardos I, Fischer S, Kumar N, Clavel T. Rhea: a transparent and modular R pipeline for microbial profiling based on $16 \mathrm{~S}$ rRNA gene amplicons. PeerJ 2017;5:e2836.

44. Glauben R, Batra A, Stroh T, et al. Histone deacetylases: novel targets for prevention of colitis-associated cancer in mice. Gut 2008;57:613-22.

45. Erben U, Loddenkemper C, Doerfel K, et al. A guide to histomorphological evaluation of intestinal inflammation in mouse models. Int J Clin Exp Pathol 2014:7:4557-76. 
46. Ganesh BP, Klopfleisch R, Loh G, Blaut M. Commensal Akkermansia muciniphila exacerbates gut inflammation in Salmonella Typhimuriuminfected gnotobiotic mice. PLoS One 2013;8:e74963.

47. Batra A, Heimesaat MM, Bereswill S, et al. Mesenteric fat - control site for bacterial translocation in colitis? Mucosal Immunol 2012;5:580-91.

48. Bolger AM, Lohse M, Usadel B. Trimmomatic: a flexible trimmer for Illumina sequence data. Bioinformatics 2014;30:2114-20.

49. Dobin A, Davis CA, Schlesinger F, et al. STAR: ultrafast universal RNAseq aligner. Bioinformatics 2013;29:15-21.

50. Anders S, Pyl PT, Huber W. HTSeq-a Python framework to work with high-throughput sequencing data. Bioinformatics 2015;31:166-9.

51. Love MI, Huber W, Anders S. Moderated estimation of fold change and dispersion for RNA-seq data with DESeq2. Genome Biol 2014;15:550.

52. Mootha VK, Lindgren CM, Eriksson KF, et al. PGC-1 $\alpha$-responsive genes involved in oxidative phosphorylation are coordinately downregulated in human diabetes. Nat Genet 2003;34:267-73.

53. Subramanian A, Tamayo P, Mootha VK, et al. Gene set enrichment analysis: a knowledge-based approach for interpreting genome-wide expression profiles. Proc Natl Acad Sci U S A 2005;102:15545-50.

54. Sturn A, Quackenbush J, Trajanoski Z. Genesis: cluster analysis of microarray data. Bioinformatics 2002;18:207-8.

55. Edgar R, Domrachev M, Lash AE. Gene expression omnibus: NCBI gene expression and hybridization array data repository. Nucleic Acids Res 2002;30:207-10.

56. Hernández-Chirlaque C, Aranda CJ, Ocón B, et al. Germ-free and antibiotic-treated mice are highly susceptible to epithelial injury in DSS colitis. J Crohns Colitis 2016;10:1324-35.

57. Bylund-Fellenius A-C, Landström E, Axelsson L-G, Midtvedt T. Experimental colitis induced by dextran sulphate in normal and germfree mice. Microb Ecol Health Dis 1994;7:207-15.

58. Ukena SN, Singh A, Dringenberg U, et al. Probiotic Escherichia coli Nissle 1917 inhibits leaky gut by enhancing mucosal integrity. PLoS One 2007;2:e1308.
59. Kredel LI, Batra A, Stroh T, et al. Adipokines from local fat cells shape the macrophage compartment of the creeping fat in Crohn's disease. Gut 2013;62:852-62.

60. Ekmekciu I, von Klitzing E, Fiebiger U, et al. Immune responses to broad-spectrum antibiotic treatment and fecal microbiota transplantation in mice. Front Immunol 2017;8:397.

61. Hansen CH, Nielsen DS, Kverka M, et al. Patterns of early gut colonization shape future immune responses of the host. PLoS One 2012;7:e34043.

62. Gury-BenAri M, Thaiss CA, Serafini N, et al. The spectrum and regulatory landscape of intestinal innate lymphoid cells are shaped by the microbiome. Cell 2016;166:1231-46.e13.

63. Gensollen T, Iyer SS, Kasper DL, Blumberg RS. How colonization by microbiota in early life shapes the immune system. Science 2016;352:539-44.

64. Lavin Y, Winter D, Blecher-Gonen R, et al. Tissue-resident macrophage enhancer landscapes are shaped by the local microenvironment. Cell 2014;159:1312-26.

65. Kitajima S, Morimoto M, Sagara E. A model for dextran sodium sulfate [DSS]-induced mouse colitis: bacterial degradation of DSS does not occur after incubation with mouse cecal contents. Exp Anim 2002;51:203-6.

66. Schaubeck M, Clavel T, Calasan J, et al. Dysbiotic gut microbiota causes transmissible Crohn's disease-like ileitis independent of failure in antimicrobial defence. Gut 2016;65:225-37.

67. Roulis M, Bongers G, Armaka M, et al. Host and microbiota interactions are critical for development of murine Crohn's-like ileitis. Mucosal Immunol 2016;9:787-97.

68. Rakoff-Nahoum S, Paglino J, Eslami-Varzaneh F, Edberg S, Medzhitov R. Recognition of commensal microflora by toll-like receptors is required for intestinal homeostasis. Cell 2004;118:229-41.

69. Pull SL, Doherty JM, Mills JC, Gordon JI, Stappenbeck TS. Activated macrophages are an adaptive element of the colonic epithelial progenitor niche necessary for regenerative responses to injury. Proc Natl Acad Sci U S A 2005;102:99-104. 